\title{
MORBIDADE DA ESQUISTOSSOMOSE E SUA RELAÇAO COM A CONTAGEM DE OVOS DE SCHISTOSOMA MANSONI EM UMA ZONA HIPERENDEMICA DO ESTADO DE MINAS GERAIS ( $\left.{ }^{*}\right)$
}

\author{
Marla Fernanda F. de Lima e COSTA (1), R. S. ROCHA (2) e Naftale KATZ (2)
}

\section{R E S U M O}

Um estudo seccional da esquistossomose foi desenvolvido em Comercinho, cidade de 1474 habitantes situada em Minas Gerais. Foram feitos exame de fezes pelo método de KATO-KATZ e exame clínico em, respectivamente, 90 e $80 \%$ da população da cidade. Foram estudados os sinais e sintomas apresentados pelos pacientes com diferentes contagens de ovos de S. mansoni, verificando-se que as alterações do tamanho $e$ da consistência do fígado estavam relacionadas à maior eliminação de ovos $(\geqslant 1000 / \mathrm{gr}$ fezes) nos pacientes com 2-14 anos de idade, mas não nos pacientes mais velhos. Os seguintes sinais clínicos foram mais frequientes nos pacientes que eliminavam ovos de $\mathbf{S}$. mansoni nas fezes, quando compa rados ao grupo controle (sem ovos do parasita nas fezes, sem história de tratamento e com reação intradérmica negativa): a) nos pacientes com 2-14 anos, sangue nas fezes, fígado palpável, aumento da consistência e aumento do tamanho dos lobos direito e esquerdo do fígado; b) nos maiores de 15 anos, presença de fígado palpável; c) em ambos os grupos etários, as esplenomegalias estiveram exclusivamente relacionadas à infecção pelo $\mathbf{S}$. mansoni.

\section{N T ROD U G AO}

Diversos Autores compararam os sinais e sintomas apresentados por pacientes infectados e presumivelmente não infectados pelo $\mathbf{S}$. man. soni e obtiveram resultados que diferiram entre as várias zonas endêmicas investigadas. $\mathrm{Em}$ algumas áreas, por exemplo, verificou-se que as dores abdominais $7,17,18$, a presença de san. gue nas fezes $3,4,7,13,18$, as hepatomegalias $2,5,8$. 16,18 e as esplenomegalias 11,16 estavam relacionadas à infecção pelo S. mansoni. Enquanto em outras zonas endêmicas, os sinais e sintomas gastrointestinais $2,5,16$, as hepatomegalias $7,12,17 \mathrm{e}$ as esplenomegalias $3,13,17$ foram observadas com igual frequiência nos pacientes infectados e nos pacientes presumivelmente não infectados pelo S. mansoni.
Outro aspecto que necessita ser mais investigado é a relação entre as manifestaçōes clínicas da esquistossomose e a contagem de ovos de S. mansoni nas fezes. Esta relação tem sido estudada por vários Autores e os resultados obtidos variam entre as diferentes zonas endèmicas pesquisadas $1,2,4,5,7,8,12,13,14,16,17,18$.

No presente trabalho serão apresentados os resultados obtidos em uma zona hiperendêmica do Estado de Minas Gerais, onde foram analisados comparativamente os sinais e sintomas apresentados pelos pacientes infectados e presumivelmente não infectados pelo S. mansoni, assim como os sinais e sintomas apresentados pelos pacientes com diferentes contagens de

(*) Trabalho financiado pelo Conselho Nacional de Desenvolvimento Cientifico e Tecnologico (CNPq) e pela Finan. ciadora de Estudos e Projetos (FINEP)

(1) Departamento de Medicina Preventiva e Social da Universidade Federal de Minas Gerais, Belo Horizonte, MG, Brasil

(2) Centro de Pesquisas "René Rachou" da Fundaçăo Oswaldo Cruz, Belo Horizonte 
COSTA, M. F. F. de L. e; ROCHA, R. S. \& KATZ, N. - Morbidade da esquistossomose e sua relação com a contagem de ovos de Schistosoma mansoni em uma zona hiperendêmica do Estado de Minas Gerais. Rev. Inst. Med. trop; São Paulo 27:66-75, 1985 .

ovos do parasita nas fezes. Esta área possuía características interessantes para este tipo de investigação, tais como a ausência de casos de malária e de leishmaniose visceral, um grande número de pacientes infectados (cerca de 900) e uma proporção relativamente alta de pacientes que eliminavam $\supseteq 1000$ ovos de $\mathbf{S}$. mansoni por grama de fezes (cerca de um quarto dos infectados )5.

\section{MATERIAL E Metodos}

\section{Area em estudo}

Este trabalho foi desenvolvido em Comercinho, cidade de 1474 habitantes situada no nordeste do Estado de Minas Gerais, onde não existe esgoto sanitário e onde somente $32,4 \%$ dos domicílios estão ligađos à rede pública de água. o hospedeiro intermediário é a Biomphalaria glabrata 6 .

\section{Exame de fezes}

Foram preparadas duas lâminas da amos: tra de fezes de cada paciente e a média do resultado obtido nas duas lâminas foi considerada como o número de ovos de $\mathbf{S}$. mansoni por grama de fezes do paciente. O método adotado foi $o$ de KATO motificado por KATZ \& col. ${ }^{9}$ e todos os exames foram realizados pelo mesmo indivíduo. Foram considerados positivos os pacientes que apresentavam ovos de S. mansoni em pelo menos uma das lâminas examinadas.

\section{Exame clínico}

Um único médico aplicou um questionário para obter informações sobre os sinais e sintomas apresentados pelos pacientes nos trinta dias que antecederam a entrevista. Os sinais e sintomas pesquisados foram os seguintes: dor abdominal (de qualquer tipo ou intensidade em qualquer quadrante do abdome), sangue nas fezes (sangue vivo em qualquer quantidade), diarréia, melena e hematêmese. Foram considerados "assintomáticos" os pacientes que não apresentavam nenhum destes sinais e sintomas.

o exame físico foi feito por outro médico, com o paciente em decúbito dorsal e em decúbito lateral direito, considerando-se palpáveis 0 fígado ou o baço detectados imediatamente abai- xo do rebordo costal com a respiração em re. pouso. Quando palpável(is), a distância máxima destes órgãos foi medida em centímetros abaixo do rebordo costal. Todas as medidas foram feitas com o paciente em decúbito dorsal e a respiração em repouso. Durante o exame, ambos os médicos desconheciam os resultados dos exames de fezes dos pacientes.

\section{Reação intradérmica}

A reação intradérmica foi feita nos pacien. tes que não apresentavam ovos de $\mathbf{S}$. mansoni no exame de fezes e que nunca haviam sido tratados com esquistossomicida(s). O antígeno foi preparado com vermes adultos da cepa LE de S. mansoni (C. P. "René Rachou"); e a leitura do teste foi feita 15 minutos após a aplicação do antigeno na região supra-escapular 15. Foram considerados negativos-1 os pacientes que não apresentavam ovos de $\mathbf{S}$. mansoni no exame de fezes e que também apresentavam reação intradérmica negativa (pápula $<1 \mathrm{~cm}^{2}$ ). Foram denominados negativos-2 aqueles sem ovos do parasita nas fezes, mas com história de tratamento específico ou com reação intradérmica positiva (pápula $\supseteq 1 \mathrm{~cm}^{2}$ ).

\section{Análise estatística}

Para avaliar a significância estatística das diferenças entre as frequiencias foi aplicado o teste do Qui quadrado com correção de YATES 19. Para as diferenças entre as médias foram aplicados o teste $t$ de STUDENT (Tabelas I, II e V) ou a análise de variância e o teste de SCHEFEE (Tabelas III e IV) ". O valor mínimo de significância considerado foi $95 \%$ $(p<0,05)$.

\section{RESULTADOS}

A Fig. 1 mostra a distribuição da população de Comercinho, segundo a faixa etária, o sexo e a condição de ter-se submetido aos exames clínico e de fezes. Foram feitos exames de fezes em $90,2 \%$ e exame clínico em $80,1 \%$ da população total da cidade. Foram examinados clinicamente 880 pacientes positivos (94\% do total de positivos) e 301 pacientes negativos (91,2\% do total de negativos com $\geq 2$ anos de idade); não foram encontrados casos positivos entre os menores de 2 anos. 
COSTA, M. F. F. de I. e; ROCHA, R. S. \& KATZ, N. - Morbidade da esquistossomose e sua relação com a contagem de ovos de Schístosoma mansoni em uma zona hiperendêmica do Estado de Minas Gerais. Rev. Inst. Med. trop. São Paulo 27:66-75, 1985 .

T A B E I A I

Sinais e sintomas apresentados pelos pacientes com 2-14 anos, segundo a positividade para o Schlstosoma mansoni

\begin{tabular}{|c|c|c|c|}
\hline Sinais e sintomas & Positivos $(\star)$ & Negativos -1 & Negativos·2 $\left(^{\star}\right)$ \\
\hline Dor abdominal & $(303) 67,6$ & (51) 45,9 & (23) 60,5 \\
\hline Diarréia & $(305) 68,1$ & $(51) 45,9$ & $(21) 55,3$ \\
\hline Sangue nas fezes & $(250) 55,8\left(^{*}\right)$ & (22) 19,8 & (10) 26,3 \\
\hline Melena & ( 2) 0,4 & ( 0$) 0,0$ & ( 0$) 0,0$ \\
\hline Hematêmese & ( 0$) 0,0$ & ( 0$) 0,0$ & ( 0 ) 0,0 \\
\hline Ascite & ( 1) 0,2 & $(0) 0,0$ & $(0) 0,0$ \\
\hline "Assintomático" & $(89) 19,9(\star)$ & $(53) 47,7$ & $(12) 31,6$ \\
\hline $\begin{array}{l}\text { TOTAL DE PACIENTES } \\
\text { Figado }\end{array}$ & $(448) 100,0$ & (111) 100,0 & (38) 100,0 \\
\hline Nāo palpável & $(206) 46,0$ & $(73) 65,8$ & (24) 632 \\
\hline Palpável & $(242) 54,0(*)$ & $(38) 34,2$ & $\begin{array}{l}(24) 63,2 \\
(14) 36,8\end{array}$ \\
\hline TOTAL & $(448) 100,0$ & (111) 100,0 & (38) 100,0 \\
\hline Palpável na LHC & $(236) 97,5$ & $(38) 100,0$ & (13)92,9 \\
\hline Palpável na LME & $(240) 99,2$ & $(38) 100,0$ & (14) 100,0 \\
\hline Tamanho médio na LHC & $(236) 3,1 \pm 1,5\left(^{*}\right)$ & $(38) 2,6 \pm 1,2$ & $\left.(13) 3,6 \pm 1,00^{*}\right)$ \\
\hline Tamanho médio na LME & $(240) 5,4 \pm 1,9\left(^{\star}\right)$ & $(38) 2,9 \pm 2,2$ & $(14) 5,4 \pm 2,1\left(^{*}\right)$ \\
\hline Consistência normal & ( 75) 31,0 & $(37) 97,4$ & ( 5$) 35,7$ \\
\hline Consistência aumentada & $(167) 69,0(*)$ & ( 1) 2,6 & $(9) 64,3(*)$ \\
\hline TOTAL & $(242) 100,0$ & (38) 100,0 & (14) 100,0 \\
\hline \multicolumn{4}{|l|}{ Baço } \\
\hline Não palpável & (419)92,5 & (111) 100,0 & (34)89,5 \\
\hline Palpável & ( 29) $6,5(*)$ & (0) 0,0 & (4) $10,5(*)$ \\
\hline TOTAL & $(448) 100,0$ & (111) 100,0 & (38) 100,0 \\
\hline
\end{tabular}

: (n) $\%$ ou média $\pm s$

(*): Diferenças estatisticamente significantes em relação aos negativos-1 (p $<0,05$ )

LHC: linha hemiclavicular

LME: linha medioesternal

Dos 1329 pacientes que se submeteram ao exame de fezes, $936(70,4 \%)$ apresentaram ovos de $\mathbf{S}$. mansoni neste exame. Cinqüenta e sete pacientes haviam sido tratados com esquistossomicida(s) e eliminavam menor número de ovos de S. mansoni nas fezes do que os pacientes nunca tratados (média geométrica $=186,8 \pm$ 4,3 e $343,2 \pm 4,8$ ovos/g fezes, respectivamente); os positivos que já haviam sido tratados foram excluídos da análise. Além disto, não foi possivel fazer a palpação abdominal em 18 mulheres ( 17 positivas e 1 negativa-2) que se encontravam nos últimos meses de gravidez.

A Tabela I mostra os sinais e sintomas apresentados pelos pacientes com 2-14 anos de idade, segundo a positividade para o $\mathbf{S}$. manso. ni. Os pacientes positivos apresentaram as seguintes diferenças em relação aos negativos-1: a) menor percentagem de casos "assintomáticos" e maior percentagem de sangue nas fezes; b) maior percentagem de fígados palpáveis $(54,0 \%$ dos positivos em relação a $34,2 \%$ dos negativos-1); c) maior tamanho médio dos lobos direito e esquerdo do figado; d) percentagem significativamente mais alta de fígados com a consistência aumentada $(69,0 \%$ dos positivos em relação a $2,6 \%$ dos negativos-1); f) presença de baço palpável (nenhum paciente negativo-1 apresentava esplenomegalia). O tamanho médio dos lobos direito e esquerdo do fígado, a percentagem de fígados com a consistência aumentada e o indice de esplenomegalias foram significativamente mais altos entre os pacientes negativos-2 do que entre os negativos-1. $E$ importante salientar que a percentagem de fígados palpáveis entre os negativos-1 diminuiu progressivamente à medida em que a idade dos pacientes aumentou $(43,8,30,4$ e $17,6 \%$, respectivamente, nos negativos $1 \mathrm{com}$ 2-4, 5.9 e 10-14 anos de idade).

A Tabela II mostra os sinais e sintomas apresentados pelos pacientes positivos, negativos-1 e negativos-2 com $\supseteq 15$ anos de idade. A percentagem de pacientes com o fígado pal. pável foi significativamente mais alta entre os positivos $(29,3 \%)$ e entre os negativos-2 $(15,9 \%)$ do que entre os negativos-1 (4,5\%). Nenhum paciente negativo-1 apresentava $\circ$ baço palpável, enquanto $6,7 \%$ dos positivos e $6,5 \%$ dos negativos-2 apresentavam esplenomegalia. A 
COSTA, M. F. F. de L. e; ROCHA, R. S. \& KATZ, N. - Morbldade da esquistossomose e sua relaçāo com a contagem de ovos de Schlstosoma mansonl em uma zona hiperendêmica do Estado de Minas Gerais. Rev. Inst. Med. trop. Sáa Paulo 27:66-75, 1985 .

T A B E L A II

Sinais e sintomas apresentados pelos pacientes com $\supseteq 15$ anos de idade, segundo a positividade para o Schistosoma manson:

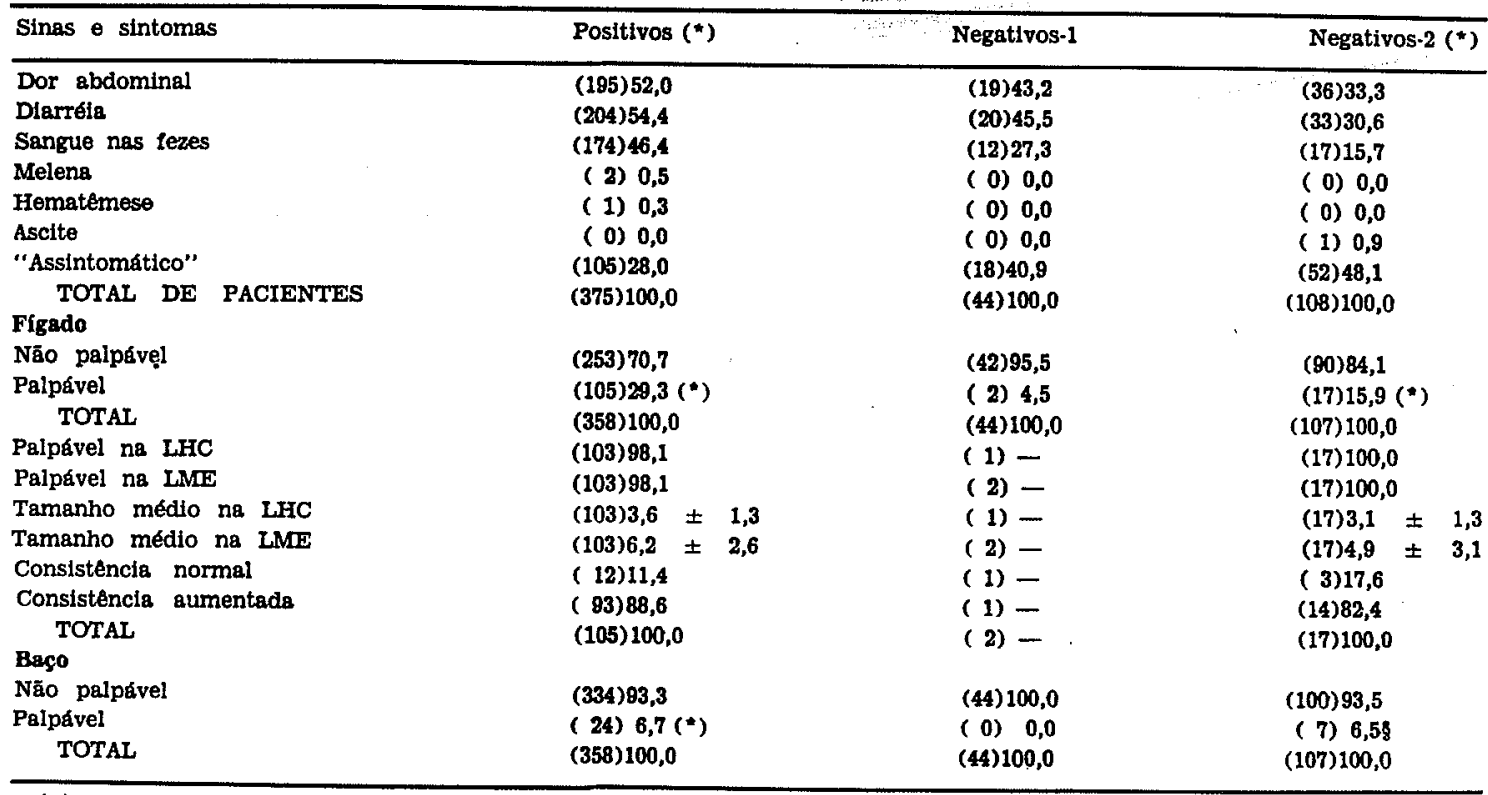

\footnotetext{
: (n) $\%$ ou média $\pm s$

(*): Diferenças estatisticamente significantes em relaçao aos negativos-1 $(p<0,05)$

- : Percentagens e médias aritméticas nåo calculadas $(n \leq 2)$

$\S$ : 3 destes pacientes haviam sido esplenectomlzados

LHC: linha hemiclavicular

LME: linha medioesternal
}

comparação do tamanho e da consistência do fígado entre os três grupos não foi feita porque só dois pacientes negativos-1 apresentavam o fígado palpável.

Nos pacientes com 2-14 anos, os seguintes sinais clínicos estiveram relacionados a maiores contagens ( $\geqslant 1000$ ovos $/ g$ ) de ovos de $\mathbf{S}$. mansoni nas fezes: presença de ffgado palpável, aumento da consistência deste órgão e maior tamanho do lobo esquerdo do fígado (Tabela III). Nos pacientes mais velhos, nenhum dos sinais e sintomas pesquisados apresentou relação com a contagem de ovos de $\mathbf{S}$. mansoni nas fezes (Tabela IV).

A Tabela $\mathrm{V}$ mostra as características do figado e do baço nos pacientes positivos com esplenomegalia. Ressaltam-se as seguintes observações: a) todos estes pacientes apresentavam o fígado palpável e com a consisténcia aumentada; b) o tamanho médio do lobo esquerdo do figado nestes pacientes foi 7,8 centímetros; c) $60,4 \%$ dos baços eram palpáveis entre 1 e 4 centímetros do rebordo costal; d) o tamanho médio do baço nesta área endêmica foi 4,7 centímetros; e) o baço com a consistência aumentada foi mais frequiente $(83,3 \%)$ nos pacientes com $\geq 15$ anos do que nos pacientes com 2-14 anos $(55,2 \%)$; $)$ o tamanho médio do baço foi maior nos pacientes com $\triangleq 15$ anos $(5,4 \mathrm{~cm})$ do que nos mais jovens $(4,0 \mathrm{~cm})$.

\section{DISCUSSAO}

Diversos Autores estudaram comparativamente os sinais e sintomas apresentados por pacientes infectados e presumivelmente não infectados pelo S. mansoni, mas os critérios adotados para a definição dos grupos "não infectados" não foram uniformes. Em algumas áreas foram considerados "não infectados" os pacientes que não apresentavam ovos de S. mansoni em um $1,4,7,8,12,16,17$ ou mais $3,5,18$ exames de fezes e em outras áreas foi levado em conta também o resultado da reação intradérmi$\mathrm{ca}^{2}$. Da mesma forma, não existe consenso 
COSTA, M. F. F. de L. e; ROCHA, R. S. \& KATZ, N. - Morbidade da esquistossomose e sua relaçäo com a contagem de ovos de Schlstosoma mansoni em uma zona hiperendêmica do Estado de Minas Gerais. Rev. Inst. Med. trop. Săo Paulo 27:66-75, 1985 .

T A B E L A III

Sinais e sintomas apresentados pelos pacientes com 2-14 anos de idade, segundo a contagem de ovos de Schistosoma mansonl nas fezes

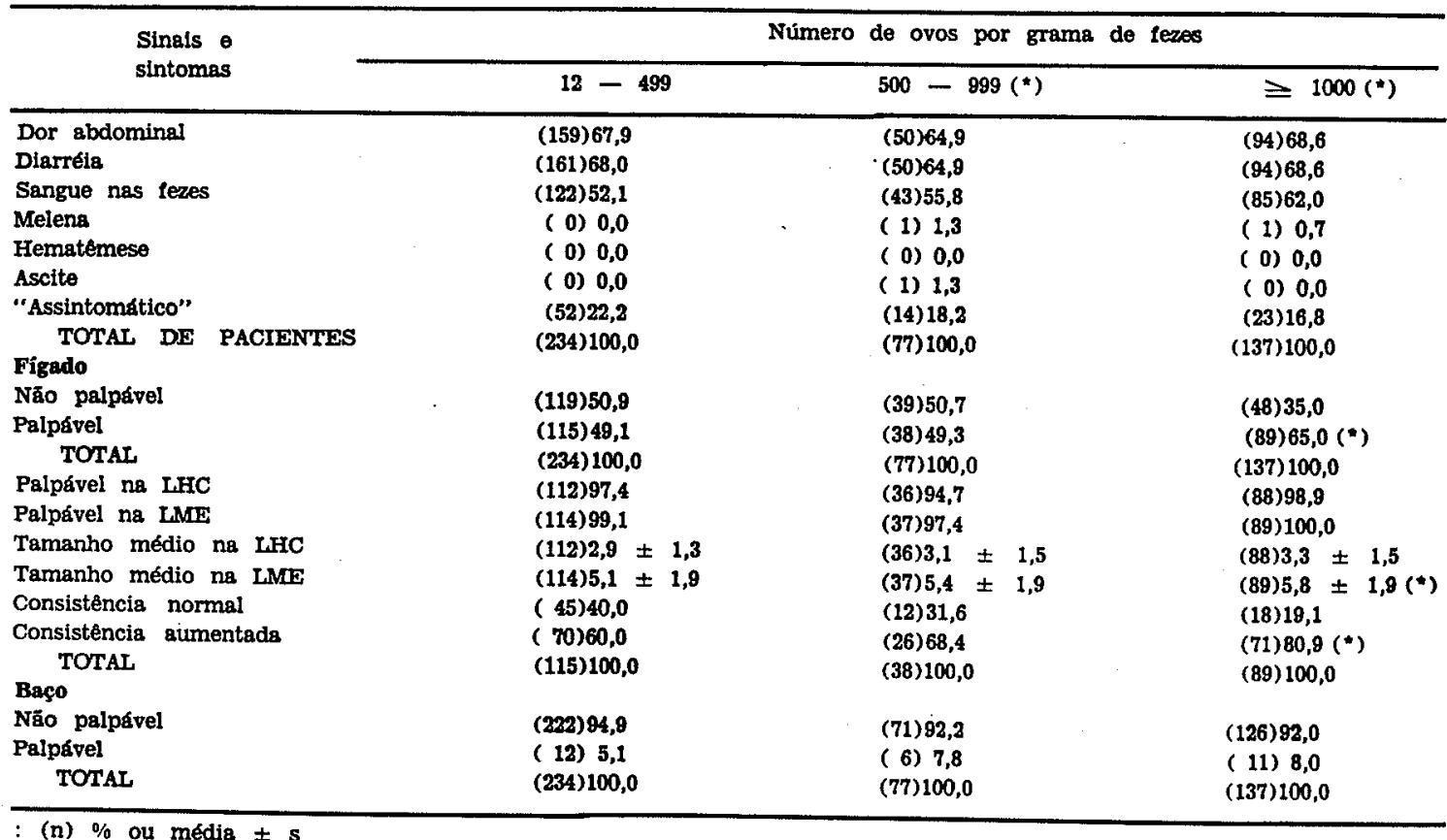

(*): Diferenças estatisticamente significante em relaçio ao grupo com $12-499$ ovos/g fezes

LHC: linhe hemiclavicular

LME: linha medioesternal

no que se refere à inclusão ou não dos pacientes tratados com esquistossomicidas no grupo presumivelmente não infectado. CLINE \& col. ${ }^{3}$ excluíram seus pacientes tratados deste grupo. ABDEL-WAHAB \& col. ${ }^{1}$ e OMER \& col. $13 \mathrm{man}$ tiveram os pacientes tratados no grupo negativo e outros autores não mencionaram esta variável $4,5,7,8,12,16,17,18$.

No presente trabalho foram considerados presumivelmente não infectados (negativos-1) os pacientes que não apresentavam ovos de S. mansoni em um exame de fezes, que nunca haviam sido tratados e que também apresentavam reação intradérmica negativa. O grupo negativo.2 (sem ovos de S. mansoni no exame de fezes, mas com reação intradérmica positiva ou com história de tratamento especifico) foi constituído para testar a validade dos critérios adotados para a definição do grupo negativo-1. Os negativos-2, de uma maneira geral, apresentaram sinais clínicos semelhantes aos dos pacientes positivos, enquanto os negativos-1 diferiram com mais frequiência de ambos. Isto indica que a ausência de ovos nas fezes, complementada pela reação intradérmica e pela história de tratamento, pode excluir grande parte dos pacientes que já apresentaram ou presumivelmente apresentam infecção pelo S. mansoni năo detectada à coproscopia.

Dentro destes critérios, o sinal clínico que mais diferenciou as crianças infectadas pelo $\mathbf{S}$. mansoni, em relação àquelas presumivelmente não infectadas, foi o aumento da consistência do fígado: a percentagem de fígados com a consistência aumentada foi 26 vezes maior nos positivos do que nos negativos-1 com 2-14 anos de idade. A percentagem de pacientes com o fígado palpável foi 1,6 e 6,5 vezes maior nos grupos positivos do que nos negativos-1 com, respectivamente, 2-14 e $\geqslant 15$ anos de idade. A presença de sangue nas fezes foi 2,8 vezes mais freqüente no grupo positivo do que no grupo negativo-1 com 2.14 anos de idade. Nos mais velhos, este sinal clínico também fol mais frequiente no grupo infectado $(46,4 \%)$ do que no grupo controle $(27,3 \%)$, mas a diferença obser- 
COSTA, M. F. F. de L. e; ROCHA, R. S. \& KATZ, N. - Morbidade da esquistossomose e sua relação com a contagem de ovos de Schistosoma mansoni em uma zona hiperendêmica do Estado de Minas Gerais. Rev. Inst. Med. trop. São Paulo 27:66-75, 1985 .

T A B E L A IV

Sinais e sintomas apresentados pelos pacientes com $\supseteq 15$ anos de idade, segundo a contagem de ovos de Schistosoma mansoni nas fezes

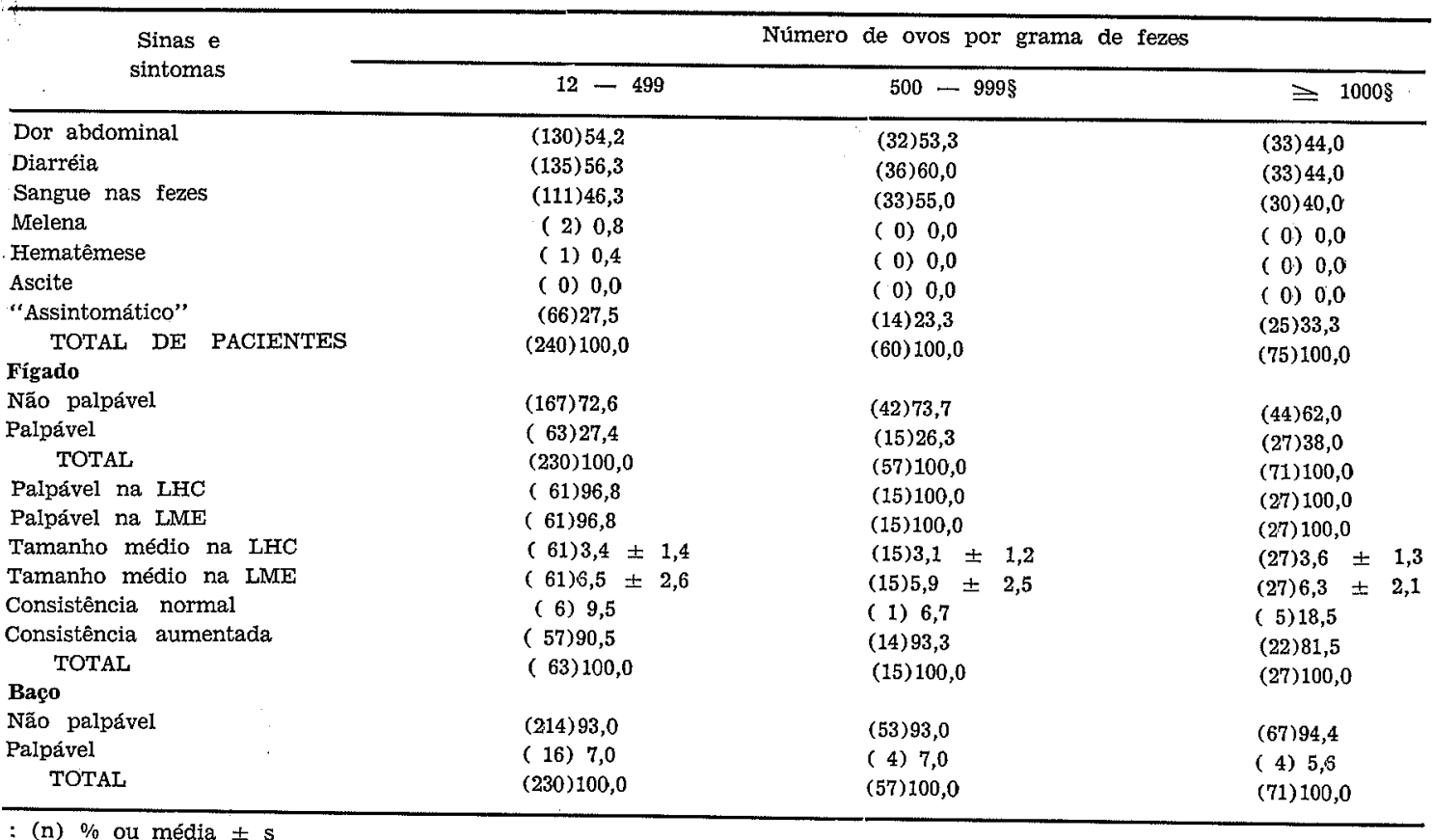

\$ : Nenhuma das diferenças em relação ao grupo com $12-499$ ovos/g fezes foi estatisticamente significante LHC: linha hemiclavicular

LME: linha medioeșternaI

vada não foi estatisticamente significante. A presença de esplenomegalia em Comercinho esteve exclusivamente relacionada à infecção pelo S. mansoni em ambos os grupos etários (nenhum paciente negativo-1 apresentou esplenomegalia).

Estudos seccionais, analisando amostras representativas de populações residentes em zonas endêmicas de $\mathbf{S}$. mansoni, têm mostrado que nestas áreas predominam as pequenas esplenomegalias e que os episódios de hematêmese ou a presença de ascite são eventos raros èm relação às manifestações gerais da esquistossomose $7,14,16$. Os resultados obtidos no presente trabalho confirmam estas observaçôes.

A relação entre as manifestações clínicas da esquistossomose e a "intensidade" da infecção pelo S. mansoni tem sido estudada por vários Autores e o resultado que mais se repete é a associação entre a maior eliminação de ovos nas fezes e a presença de hepatomegalia. De fato, observou-se em dez diferentes locali- dades que as hepatomegalias eram mais frequientes no conjunto da população $2,4,16,18$ ou nas crianças 1,5,8,12,14 (quando estas eram ana. lisadas separadamente) que eliminavam maior número de ovos de S. mansoni nas fezes e em quatro localidades $7,10,17$ verificou-se que a presença de hepatomegalia não estava relacionada à maior ou menor eliminação de ovos nas fezes. Em relação às esplenomegalias, verificouse em cinco zonas endêmicas que os pacientes com esta característica eliminavam maior número de ovos nas fezes $2,4,5,12,16$, mas em cinco outras localidades (em duas das quais a malá ria ocorria endemicamente) verificou-se que a contagem de ovos de $\mathbf{S}$. mansoni não diferia entre os pacientes que apresentavam ou não esplenomegalia 2,10,14,17. A associação entre a maior eliminação de ovos de $\mathbf{S}$. mansoni e a presença de dores abdominais ou de sangue nas fezes foi demonstrada em, respectivamente, duas áreas endêmicas do Quênia ${ }^{16,17} \mathrm{e} \mathrm{em}$ duas áreas do Sudão ${ }^{13}$ e do Egito 1 ; mas em cinco outras áreas situadas em Santa Lúcia ;, 
COSTA, M. F. F. de L. e; ROCHA, R. S. \& KATZ, N. - Morbidade da esquistossomose e sua relaçāo com a contagem de ovos de Schistosoma mansoni em uma zona hiperendêmica do Estado de Minas Gerais. Rev. Inst. Med. trop. Sāo Paulo $27: 66-75,1985$.

T A B E L A V

Características do fígado e do baço nos pacientes com esplenomegalia, segundo a faixa etária

\begin{tabular}{|c|c|c|c|}
\hline \multirow{2}{*}{$\begin{array}{l}\text { Características do } \\
\text { fígado e do baço }\end{array}$} & \multicolumn{2}{|c|}{ Faixas etárias } & \multirow{2}{*}{ TOTAL } \\
\hline & 2-14 anos & $\supseteq 25$ anos (*) & \\
\hline Fígado & & . & \\
\hline Não palpável & (0) 0,0 & (0) 0,0 & (0) 0,0 \\
\hline Palpável & (29) 100,0 & (24) 100,0 & (53) 100,0 \\
\hline TOTAL & (29) 100,0 & (24) 100,0 & (53) 100,0 \\
\hline Palpável na LHC & (29) 100,0 & (23) 95,8 & (52) 98,1 \\
\hline Palpável na LME & $(29) 100,0$ & (24) 100,0 & (53) 100,0 \\
\hline Tamanho na LHC & (29) $3,1 \pm 1,4$ & (23) $3,7^{\circ} \pm 1,7$ & (52) $3,3 \pm 1,6$ \\
\hline Tamanho na LME & (29) $7,4 \pm 1,7$ & (24) $8,3 \pm 2,8$ & (53) $7,8 \pm 2,3$ \\
\hline Consistência normal & $(0) 0,0$ & $(0) 0,0$ & $(0) 0,0$ \\
\hline Consistência aumentada & (29) 100,0 & (24) 100,0 & (53) 100,0 \\
\hline TOTAL & (29) 100,0 & (24) 100,0 & (53) 100,0 \\
\hline \multicolumn{4}{|l|}{ Baço } \\
\hline \multicolumn{4}{|l|}{ Tamanho em centimetros } \\
\hline$<1$ & ( 2) 6,9 & ( 0$) 0,0$ & ( 2) 3,8 \\
\hline $1-4$ & (18) 62,1 & (14) 58,3 & $(32) 60,4$ \\
\hline $5-9$ & ( 8$) 27,6$ & ( 6$) 25,0$ & (14) 26,4 \\
\hline$\supseteq 10$ & ( 1 ) 3,4 & ( 4$) 16,7$ & ( 5) 9,4 \\
\hline TOTAL & $(29) 100,0$ & (24) 100,0 & (53) 100,0 \\
\hline Média & $4,0 \pm 2,3$ & $5,4 \pm 3,4\left(^{*}\right)$ & $4,7 \pm 2,9$ \\
\hline \multicolumn{4}{|l|}{ Consistência } \\
\hline Normal & (13) 44,8 & ( 4) 16,7 & (17) 32,1 \\
\hline Aumentada & (16)55,2 & $(20) 83,3\left(^{*}\right)$ & $(36) 67,9$ \\
\hline TOTAL & (29) 100,0 & (24) 100,0 & (53) 100,0 \\
\hline
\end{tabular}

: (n) $\%$ ou média $\pm s$

$\left(^{*}\right)$ : Diferenças estatisticamente significantes $€ m$ relaçäo à faixa de $2-14$ anos $(p<0,05)$

LHC: linha hemiclavicular

LME: linha medioesternal

na Etiópia 7,8 e no Brasil 10 verificou-se que os sinais e sintomas gastrintestinais independiam da contagem de ovos do parasita nas fezes.

No presente trabalho, a presença de fígado palpável, o aumento do tamanho do lobo esquerdo e o aumento da consistência do fígado estiveram relacionados à maior eliminação de ovos de $S$. mansoni ( $\Rightarrow 1000$ ovos $/ g$ fezes) nos pacientes com 2-14 anos de idade, mas não a presença de esplenomegalia e os sinais e sintomas gastrintestinais. Nos pacientes mais velhos ( tomas pesquisados esteve relacionado ao número de ovos de $\mathbf{S}$. mansoni nas fezes. É interessante observar que em três outras áreas endêmicas do Egito 1, do Brasil 12 e de Uganda 14 verificou-se que as alteraçōes do fígado estavam também associadas à maior eliminação de ovos nos pacientes jovens, mas não nos mais velhos, e não existe até o momento uma explicação plausivel para este fenômeno. A análise seccional é limitada também para explicar porque a contagem de ovos de $\mathbf{S}$. mansoni não diferiu entre os pacientes que apresentavam ou não esplenomegalia. Eossível que só através de estudos prospectivos estes dois fenômenos se tornem melhor compreendidos.

Em resumo, os resultados obtidos no presente trabalho levam às seguintes conclusões: a) nesta área endêmica, a presença de sangue nas fezes, a presença de fígado palpável, o maior tamanho dos lobos direito e esquerdo do fígado e o aumento da consistência deste órgão estiveram relacionados à infecção pelo $\mathbf{S}$. mansoni nos pacientes com 2-14 anos de idade; b) nos pacientes com $\supseteq 15$ anos, a presença de fígado palpável esteve mais fortemente associada à infecção pelo $\mathbf{S}$. mansoni do que nos mais jovens; c) em ambos os grupos etários, as esplenomegalias estiveram exclusivamente associadas à infecção pelo S. mansoni; d) as altera ções do tamanho e da consistência do fígado estiveram relacionadas à maior eliminação de ovos de S. mansoni nas fezes dos pacientes com 2-14 anos de idade, sendo que contagens de ovos inferiores a 1000/g fezes não implicaram em di. ferenças nos sinais e sintomas pesquisados; e) os sinais e sintomas apresentados pelos pa- 
COSTA, M. F. F. de L. e; ROCHA, R. S. \& KATZ, N. - Morbidade da esquistossomose e sua relaçäo com a contagem de ovos de Schistosoma mansoni em uma zona hiperendêmica do Estado de Minas Gerais. Rev. Inst. Med. trop. São Paulo 27:66-75, 1985 .

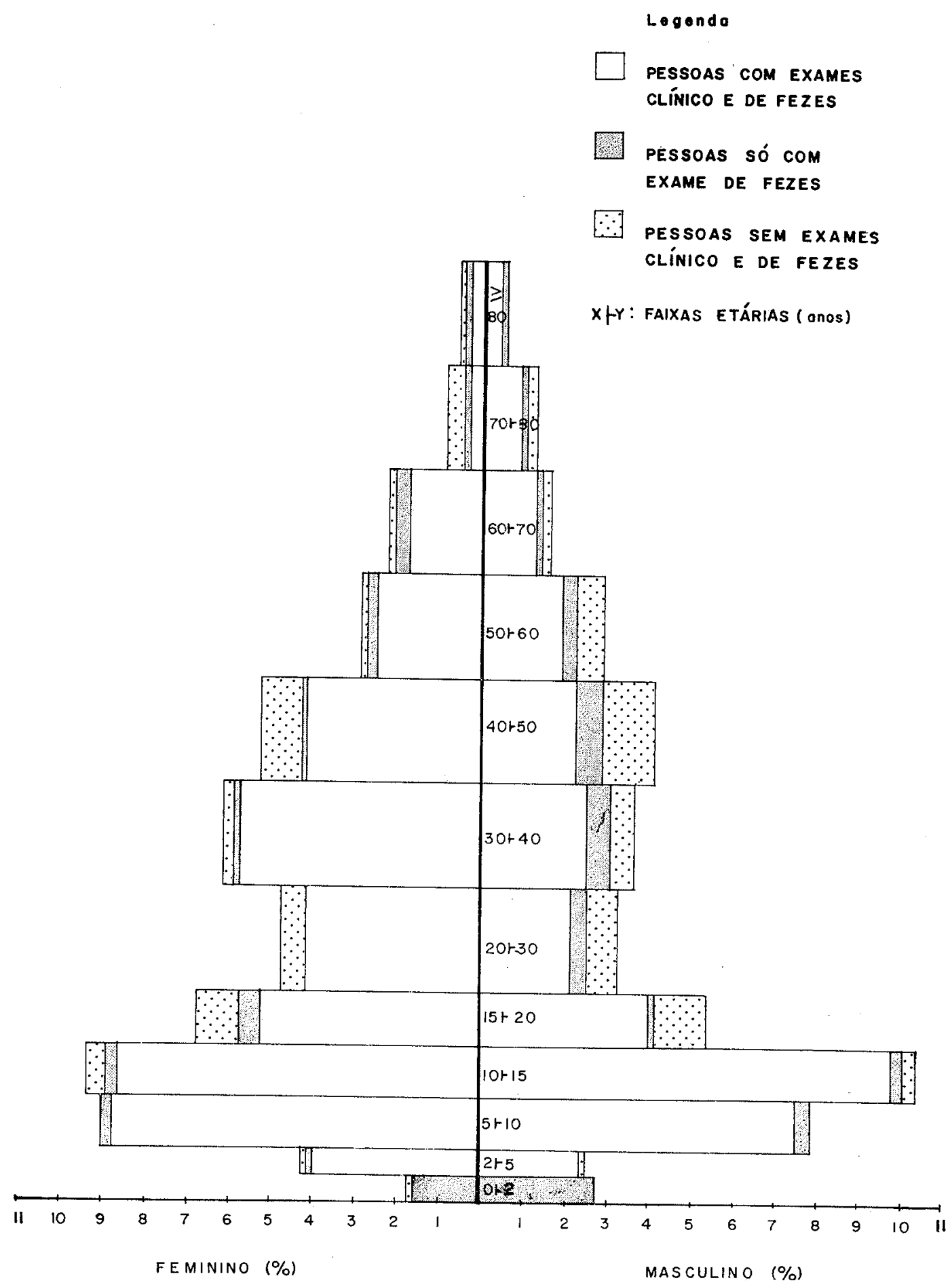

Fig. 1 - Distribuição da população segunđo a faixa etária, o sexo e a condição de ter-se submetido aos exames clínico e de fezes - Comercinho (1981) 
COSTA, M. F. F. de L. e; ROCHA, R. S. \& KATZ, N. - Morbidade da esquistossomose e sua relaçăo com a contagem de ovos de Schistosoma mansoni em uma zona hiperendêmica do Estado de Minas Gerais. Rev. Inst. Med. trop. São Paulo $27: 66-75,1985$.

cientes mais velhos não estiveram relacionados à. maior ou menor eliminação de ovos de $\mathbf{S}$. mansoni nas fezes.

\section{SUIMIMARY}

\section{Schistosomiasis morbidity and it's relation} with Schistosoma mansoni EGG count in an hyperendemic area of the Minas Gerais State

A cross-sectional survey on schistosomiasis was done in Comercinho (Minas Gerais State, Brazil), a town with 1474 inhabitants. Quantitative stool egg count (KATO KATZ technique) and clinical examination were done on 90 and $80 \%$ of the total population, respectively. Signs and symptoms presented by patients with different S. mansoni egg counts were compared and the alterations of the liver size and consistency were related to higher egg excretion ( $\supseteq 1000 / \mathrm{g}$ feces) in 2-14 year age group, but not in older patients. The following clinical signs were more frequent in patients that excreted S. mansoni eggs when compared to the control group (no S. mansoni eggs in the feces, absence of previous specific treatment and negative intradermal test): a) in patients from 2-14 years of age, blood in the feces, palpable liver, hardening of the liver and enlargement of the liver right and left lobes; b) in above 15 year old individuals, palpable liver; c) in both age groups, the splenomegalies were exclusively related to $\mathbf{S}$. marsoni infection.

\section{AGRADECIMENTOS}

Os Autores agradecem aos senhores Gercy Souza Morais, Pedro Coura e José Ribeiro, pela colaboração técnica que prestaram a este trabalho.

\section{REFERENCIAS BIBLIOGRÁFICAS}

1. ABDEL-WAHAB, M. F.; STRICKLAND, G. T.; EL SAFIY, A.; AHMED, L.; ZAKARIA, S.; EL KADY, N. \& MAHMOUD, S. - Schistosomiasis mansoni in an Egyptian village in the Nile Delta. Am. J. Trop. Med. Hyg. 29: 868-874, 1980.

2. BARBOSA, F. S. - Morbidade da esquistossomose. Rev. Bras. Malariol. Doenças Trop. N.` especial: 3-159, 1960 .

3. CLINE, B. L.; RIMZO, W. T.; HIATT, R. S. KNIGHT, W. B. \& BERRIOS-DURAN, I, S. - Morbidity from
Schistosoma mansoni in a Puerto Rican community; a population-based study. Am. J. Trop. Med. Hyg. 26: $109-117,1977$.

4. CONCEIÇÃO, M. J. - Morbidade da esquistossomose mansoni em uma comunidade rural de Minas Gerais. [Tese, mestrado]. Universidade Federal do Rio de Janeiro, Rio de Janeiro, 1975, 91 p.

5. COOK, J. A.; EIAKER, S. T.; WARREN, IK. S. \& JORDAN, P. - A controlled study of morbidity from schistosomiasis mansoni in St, Lucian children, based on quantitative egg excretion. Am. J. Trop. Med. Hyg. 23: $625-633,1974$.

6. COSTA, M. F. F. L. - Estudo clínico-epidemiológico da esquistossomose mansoni em Comercinho, Minas Gerais (1974/1981). [Tese, doutorado]. Universidade Federal de Minas Gerais, Belo Horizonte, 1983, 207 p.

7. HIATT, R. S. - Morbidity from Schistosoma mansoni infections; an epidemiologic study based on quantitative analysis of egg excretion in two highlands Ethiopian villages. Am. J. Trop. Med. Hyg. 25: 808817, 1976 .

8. HIATT, R. S. \& GEBRIN-MEDHIN, M. - Morbidity from Schistosoma mansoni infections; an epidemiologic study based on quantitative analysis of egg excretion in Ethiopian children. Am. J. Trop. Med. Hyg. 26: 473 $481,1977$.

9. KATZ, N.; CHAVES, A, \& PELLEGRINO, J. - A simple device for quantitative stool thick-smear technique in schistosomiasis mansoni. Rev. Inst. Med. trop. São Paulo 14: $397-400$, 1972.

10. KATZ, N. \& ZICKER, F. - Correlation between symptomatology and intensity of Schistosoma mansoni infection in inhabitants from endemic areas in Minas Gerais state-Brazil. Brasillia Méd. 11: 55-59, 1975.

11. KLEINBAUM, D. G. \& KUPPER, L. L. - Applied Regression Analysis and Other Multivariable Methods. Massachussets, Duxbury Press, 1978.

12. LEHMAN Jr., J. S.; MOTT, K. E.; MORROW Jr., R. H.; MUNIZ, T. M. \& BOYER, M. H. - The intensity and effects of infections with Schistosoma mansoni in a rural community in Northeast Brazil. Am. $J$. Trop. Med. Hyg. 25: 285-294, 1976.

13. OMER, A. H. S.; HAMILTON, P. J. S.; MARSHALL, T. F. C. \& DRAPER, C. C. - Infection with Schistosoma mansoni in the Geriza area of the Sudan. J. Trop. Med. Hyg. 79: 151-157, 1976.

14. ONGOM, V. L. \& BRADLEY, D. J. - The epidemiology and consequences of Schistosoma mansoni infection in the West Nile, Uganda. I. Field studies of a community at Panyagoro. Trans. R. Soc. Trop. Med. Hyg. 66: 835-851, 1972.

15. PELLEGRINO, J. \& MEMORIA, J. M. P. - A reação intradérmica na esquistossomose mansoni. III - In. 
COSTA, M. F. F. de L. e; ROCHA, R. S. \& KATZ, N. - Morbidade da esquistossomose e sua relação com a contagem de ovos de Schistosoma mansoni em uma zona hiperendêmica do Estado de Minas Gerais. Rev. Inst. Med. trop. São Paulo 27:66-75, 1985.

fluência da idade, sexo, cor e local da reação. Rev. Inst. Med. trop. São Paulo 2: 218-223, 1960.

16. SIONGOK, T. K. A.; MAHMOUD, A. A. F.; OUMA, J. H.; WARREN, K. S.; MULLER, A. S.; HANDA, A. K. \& HOUSER, H. B. - Morbidity in schistosomiasis mansoni in relation to intensity of infection; study of acommunity in Machakos, Kenya. Am. J. Trop. Med. Hyg. 25: 273-284, 1976.

17. SMITH, D. H.; WARREN, K. S. \& MAHMOUD, A. A. F. - Morbidity in schistosomiasis mansoni in relation to intensity of infection; study of a community in Kisumi, Kenya. Am. J. Trop. Med. Hyg. 28: 220-229, 1979.

18. SOUZA, S. I. - Variáveis epidemiológicas na esquistossomose mansoni. Universidade Federal da Bahia, Salvador, 1973, 80 p. [Tese, Professor Assistente].

19. SPIEGEL, M. R. - Estatística. 10 ed. São Paulo, Ėd. Mc Graw-Hill do Brasil Ltda., 1976.

Recebido para publicaçāo em $7 / 5 / 1984$. 